Disclosure of Interests: Cristina Solé-Marcé: None declared, Ana Maria Alvarez-Rios: None declared, Teresa Moline: None declared, Berta Ferrer: None declared, Josep Ordi-Ros: None declared, Josefina Cortés-Hernández Grant/research support from: GSK, Speakers bureau: GSK DOI: 10.1136/annrheumdis-2019-eular.7999

\section{THU0211 EVOLUTION OF KIDNEY ANTIBODY SECRETING CELLS MOLECULAR SIGNATURE IN LUPUS PATIENTS WITH ACTIVE NEPHRITIS UPON IMMUNOSUPPRESSIVE THERAPY}

Etienne Crickx ${ }^{1,2}$, Farah Tamirou ${ }^{3,4}$, Tessa Huscenot ${ }^{5}$, Nathalie CostedoatChalumeau $^{6}$, Marion Rabant ${ }^{7}$, Alexandre Karras ${ }^{8}$, Ailsa Robbins ${ }^{9}$, Tatiana Fadeev ${ }^{1}$, Véronique Le Guern ${ }^{6}$, Philippe Remy ${ }^{10}$, Aurélie 'Hummel ${ }^{11}$ Bertrand Godeau ${ }^{2}$, Bernard Lauwerys ${ }^{3,4}$, Jean-Claude Weill ${ }^{1}$, ClaudeAgnès Reynaud ${ }^{1}$, Frederic Houssiau ${ }^{3,4}$, Matthieu Mahévas ${ }^{1,2} .{ }^{1}$ Institut Necker Enfants Malades, INSERM U1151/CNRS UMS 8253, Paris, France; ${ }^{2}$ Hôpital Henri-Mondor (APHP), Department of Internal Medicine, Créteil, France; ${ }^{3}$ Cliniques Universitaires Saint-Luc, Service de Rhumatologie, Brussels, Belgium; ${ }^{4}$ Université de Louvain, Pôle de Pathologies Rhumatismales Inflammatoires et Systémiques, Brussels, Belgium; ${ }^{5}$ Hôpital Lariboisière (APHP), Department of Internal Medicine, Paris, France; ${ }^{6}$ Cochin Hospital (APHP), Internal Medicine Department, Paris, France; ${ }^{7}$ Necker Hospital (APHP), Pathology Department, Paris, France; ${ }^{8}$ Hôpital Européen Georges-Pompidou (APHP), Department of Nephrology, Paris, France; ${ }^{9}$ Robert Debré Hospital, Department of Internal Medicine, Reims, France; ${ }^{10}$ Hôpital Henri-Mondor (APHP), Department of Nephrology, Créteil, France; ${ }^{11}$ Necker Hospital (APHP), Nephrology Department, Paris, France

Background: Pathogenic antibody secreting cells (ASC) have been identified in the kidney of SLE-prone mice, but are poorly characterized in human lupus nephritis (LN). We hypothesized that long-lived plasma cells may contribute to the failure of immunosuppressive therapy in refractory patients.

Objectives: To characterize and compare the single cell molecular signature of ASC in kidney and urine from patients with active $L N$, either untreated or after immunosuppressive therapy failure.

Methods: We included patients with biopsy proven active LN from 4 centers and meeting the ACR revised classification criteria for SLE diagnosis. Renal biopsies were scored according to 2003 International Society of Nephrology/Renal Pathology Society (ISN/RPS) classification, and stained with anti-CD138 to visualize ASC. ASC were single cell sorted as CD3-/ CD14-/CD16-/CD27 $7^{\text {high }} / C D 38^{\text {high }}$ cells. Single-cell gene expression profiling was performed by multiplex RT-PCR using Fluidigm Dynamic Arrays. We used a set of genes derived from a previous transcriptomic analysis of human splenic and bone marrow ASC to distinguish the process of ASC maturation from plasmablast $(\mathrm{PB})$ to long-lived PC. We also studied ASC transcriptional program from urine of untreated LN patients at diagnosis and after 3 and 6 months of a prospective follow up during induction therapy (Plasmo-Lup study)

Results: Immunohistochemistry stainings on kidney biopsies from both untreated $(\mathrm{N}=15)$ and refractory patients $(\mathrm{N}=6)$ showed infiltrates of CD138+ ASC mainly located in the interstitium, particularly in untreated patients. Single cell molecular signature of kidney ASC from 3 untreated patients with class IV LN revealed that these cells were mostly PB expressing multiple genes linked with cell division, and PC without longlived genes expression. This contrasted with ASC signature from 3 patients with active $\mathrm{LN}$ and mycophenolate mofetil (MMF) failure that expressed long-lived PC genes and no proliferative genes. Primary component analysis of 170 single-cells showed clustering of ASC from MMF treated patients with long-lived bone marrow PC from healthy donors that were distinct from PB/PC from untreated patients (Figure 1). A PB signature was observed in urine ASC at diagnosis, similar to their kidney counterpart. The concentration of ASC in urine in 22 untreated patients correlated with ISN/RPS classification, with higher concentration in class IV patients $(\mathrm{p}<0.01)$.

Conclusion: These results suggest that PB infiltrate kidney of untreated LN patients, and that kidney long-lived PC may contribute to the failure of immunosuppressive therapy.

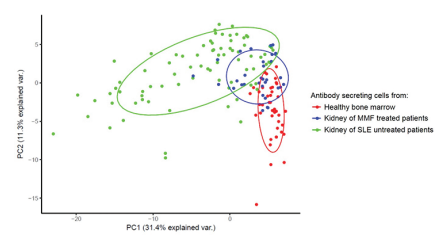

Abstract THU0211 - Figure 1. Single cell principal component analysis of 170 individual cells analyzed for the expression of 20 genes. Each dot represents a single cell.

Acknowledgement: This work was supported by Foreum Disclosure of Interests: None declared

DOI: 10.1136/annrheumdis-2019-eular.4739

\section{THU0212 BARICITINIB-ASSOCIATED CHANGES IN GLOBAL GENE EXPRESSION DURING A 24-WEEK PHASE 2 CLINICAL SLE TRIAL DESCRIBE A MECHANISM OF ACTION THROUGH INHIBITION OF JAK/STAT AND IFN RESPONSIVE GENE EXPRESSION}

Thomas Dörner ${ }^{1}$, Yoshiya Tanaka ${ }^{2}$, Michelle A Petri $^{3}$, Josef S. Smolen ${ }^{4}$ Ernst Dow ${ }^{5}$, Richard E Higgs ${ }^{5}$, Guilherme Rocha ${ }^{5}$, Robert Benschop ${ }^{5}$, Maria Silk ${ }^{5}$, Stephanie de Bono ${ }^{5}$, Robert Hoffman ${ }^{5} .{ }^{1}$ Charite Universitätsmedizin Berlin and Deutsches Rheumaforschungszentrum (DRFZ), Berlin, Germany; ${ }^{2}$ Univ of Occupational and Environmental Health, Kitakyushu, Japan; ${ }^{3}$ Johns Hopkins Univ School of Medicine, Baltimore, United States of America; ${ }^{4}$ Medical Univ of Vienna, Vienna, Austria; ${ }^{5}$ Eli Lilly and Company, Indianapolis, United States of America

Background: Baricitinib (bari) is an oral selective Janus kinase (JAK) 1 and JAK2 inhibitor. In the Phase 2 study, JAHH (NCT02708095), treat ment with bari resulted in significant improvements in patients (pts) with active systemic lupus erythematosus (SLE) receiving standard background therapy, compared with placebo (PBO). ${ }^{1}$

Objectives: To use global gene expression to characterize baseline (BL) gene expression among SLE pts in JAHH and to describe the mechanism of action (MoA) of bari during treatment in active SLE.

Methods: A total of 314 pts were randomized 1:1:1 to PBO, bari 2-mg, or bari 4-mg once daily for 24 weeks (Wks) in JAHH. RNA isolated from whole blood at $\mathrm{BL}$ and Wks 2, 4, 12, and 24 was analyzed using HTA2.0 arrays, possessing over 925,000 specific individual probes. Data were summarized to transcript level and analyzed using a mixed effects model on a log2 transformed response with multiplicity correction.

Results: Baseline gene expression analysis comparing healthy controls to SLE pts revealed elevated expression of genes involved in innate and adaptive immune pathways and biologic processes thought to be key to SLE pathogenesis. Baseline findings from the ILLUMINATE trial of tabalu mab (1760 SLE pts at $\mathrm{BL})^{2}$ were independently replicated, including individual gene-specific changes in the SLE populations versus controls. Treatment-induced changes were predominantly observed in the bari 4-mg versus PBO group at early time points (Wks 2 and 4; bari 4-mg > 2$\mathrm{mg}$ ). Statistically significant changes were observed for type I and II IFN responsive genes, and in the JAK/signal transducer and activator of transcription (STAT) canonical and noncanonical signaling pathways, in the bari 4-mg versus PBO group comparisons. There were biologically notable and statistically significant bari-associated decreases in gene expression from $\mathrm{BL}$, compared to $\mathrm{PBO}$, for interleukin (IL)-3, -5, -6, -7, and granulocyte macrophage colony-stimulating factor pathways, attributable to inhibition of JAK/STAT signaling.

Conclusion: These results build upon previous studies of gene expression in ILLUMINATE, comparing SLE at $\mathrm{BL}$ with healthy controls; they advance our understanding of lupus pathogenesis, and further delineate the MoA of bari in SLE. These data independently confirm the marked elevations of specific type I and II IFN genes at BL in clinical trial pts on standard of care treatment. Changes in gene expression with bari impacted IFN responsive genes as well as JAK/STAT signaling pathways. Interpretation of results is limited by sample size and statistical power, and presence of concomitant medications. Importantly, gene expression may not translate directly to protein expression; however, ongoing proteomic studies (including specific assays for type I IFN, IFN- $\gamma$, IL-12, IL-17, and IL-23) will help extend these gene expression results and further describe the MoA of bari in SLE.

REFERENCES:

[1] Wallace D, et al. Lancet. 2018;392:222-231

[2] Hoffman R, et al. Arthritis Rheumatol. 2017;69:643-654 